${ }^{1}$ Department of Prosthodontics and Periodontics, Periodontics Division, Piracicaba Dental School, University of Campinas, Piracicaba, SP, Brazil.

${ }^{2}$ Department of Dentistry, University of Araras, Araras, SP, Brazil

${ }^{3}$ Department of Periodontics, Paulista University, São Paulo, SP, Brazil.
Corresponding author:

Renato Corrêa Viana Casarin

Department of Prosthodontic and Periodontics

Avenida Limeira, 901 - Areião

Piracicaba, ZC 13414-903

email: rcasarin@unicamp.br phone: (19) 2106-5301

Received: October 15, 2020

Accepted: December 30, 2021

\section{Influence of} rs6667202 SNP on Interleukin-10 levels in the gingival fluid of patients with periodontitis grade $\mathrm{C}$

\author{
Camila Schmidt Stolf ${ }^{1}$ (iD), Tiago Taiete ${ }^{1,2}$ iD, Márcio \\ Zaffalon Casati ${ }^{1,3}$ iD, Enílson Antônio Sallum ${ }^{1}$ (iD, \\ Francisco Humberto Nociti Júnior ${ }^{1}$ iD, Karina Gonzales \\ Silvério Ruiz ${ }^{1}$ (ID), Renato Corrêa Viana Casarin 1,* iD
}

Grade $\mathrm{C}$ periodontitis in youngers is characterized by a severe form of periodontitis, and IL10 rs6667202 single nucleotide polymorphism (SNP) has been described as an important feature in this disease etiology. Aim: This study aimed to evaluate, in vivo, the functionality of IL10 rs6667202 SNP on IL-10 gingival fluid levels. Methods: Thirty patients with Perio4C were selected, 15 with the IL 10 AA genotype (rs6667202) and 15 with AC/CC genotypes. The gingival fluid was collected from two sites with probing depth $\geq 7 \mathrm{~mm}$ and bleeding on probing, and two healthy sites. The IL-10 concentration was determined by Luminex/MAGpix platform. Results: In deep pockets, the IL 10 AA genotype presented a lower concentration of IL-10 when compared with AC or CC genotypes $(p<0.05)$. In shallow pockets, no difference between groups was seen ( $p>0.05$ ). Conclusion: IL10 rs6667202 SNP decreases the production of IL-10 in crevicular fluid, potentially affecting this disease progression.

Keywords: Aggressive periodontitis. Interleukin-10. Polymorphism, single nucleotide. 


\section{Introduction}

Periodontitis is an inflammatory disease of multifactorial etiology, triggered by the host's immune-inflammatory response to periodontopathogens present in the biofilm. It is clinically characterized by bone destruction and loss of conjunctive insertion, and if untreated, lead to tooth loss ${ }^{1}$. Periodontitis Stage 3 or 4, Grade C (Perio4C), previously named aggressive periodontitis ${ }^{2,3}$, is a severe form of periodontitis, affecting young systemically healthy individual ${ }^{4}$. As additional characteristics, it presents a rapid rate of progression, familial aggregation of cases, and poor response to traditional therapeutic approaches ${ }^{4-8}$, leading to difficulties in the clinical management and early edentulism ${ }^{9}$. These patients' host response presents a hyperinflammatory profile, with an imbalance between the release of pro and anti-inflammatory cytokines after the activation of inflammatory cells by periodontopathogens ${ }^{10-12}$.

The host's immune-inflammatory response is influenced by genetic factors, which determine different susceptibility profiles to the diseases' development. The different allelic variants, such as the presence of a single nucleotide polymorphism (SNP), may predispose the development of periodontitis by impacting the structure, function, or production of proteins ${ }^{13-15}$.

Previous studies demonstrated an association between the IL10 rs6667202 SNP (C>A) located in the IL10 gene and the Perio4C in a Brazilian and a pooled German-Austrian sample $e^{16-17}$. The ancestral $C$ allele was detected in a lower frequency in Perio4C patients when compared to individuals with periodontal health and chronic periodontitis, making it a potential protector against the occurrence of this disease ${ }^{16-17}$. This protective character could be explained by Interleukin-10 (IL-10) action, once this cytokine presents regulatory and anti-inflammatory properties, reducing the expression of pro-inflammatory cytokines such as interleukin $1 \beta$ (IL-1 $\beta$ ) and tumor necrosis factor a (TNF-a). Given its function, its greater or lesser expression is capable of modulating disease severity ${ }^{18-19}$. However, up to date, none functional analysis has been done.

Since rs6667202 SNP is in the upstream genic region of the $/ \mathrm{L} 10$ gene (1.3 kb region), a regulatory region, it can be speculated that it may impact the expression of IL-10 cytokine. This possibility is indirectly supported by other previous studies, which demonstrated that Perio4C patients had reduced levels of IL-10 in gingival crevicular fluid (GCF) and serum when compared to other periodontal profiles ${ }^{11,20-22}$. Casarin et al. ${ }^{20}$ (2010) demonstrated a lower production of IL-10 in the gingival crevicular fluid (GCF) in moderate and deep pockets of patients diagnosed with Perio4C when compared with similar sites of individuals with chronic periodontitis.

However, these findings were not based on genetic characteristics. It is impossible to determine whether there is a direct relationship between the presence of the polymorphism and differences in cytokine production. This type of relationship has been demonstrated for a pro-inflammatory cytokine, interleukin 6 (IL-6), by Nibali et al. ${ }^{15}$ (2013). In this study, the authors reported that Perio4C patients with rs2069827 and rs2069825 IL6 SNPs had higher levels of IL-6 when compared with Perio4C negative for both SNPs patients ${ }^{15}$. 
Therefore, this study aimed to evaluate, in vivo, the functionality of IL 10 rs6667202 SNP on GCF levels, that is, assess whether IL10 rs6667202 SNP - AA genotype - may predispose an altered production of this anti-inflammatory marker in patients with Perio4C when compared to those with AC or CC genotypes.

\section{Material and Methods}

\section{Patient Selection}

This study was approved by the Piracicaba Dental School, University of Campinas Research Ethics Committee (number 58679416.4.0000.5418). For this cross-sectional study, 30 subjects were selected from Taiete's et al. ${ }^{16}$ (2019) study population, which determined the IL-10 genotyping for 200 patients diagnosed with Perio $4 \mathrm{C}$ and 200 healthy patients. All selected patients signed an informed consent form to participate in this research, and during its execution, the principles of the Declaration of Helsinki were followed.

The 30 selected subjects were allocated to:

SNP + Group ( $n=15)$ : Perio4C with AA genotype (rs6667202).

SNP - Group ( $n=15)$ : Perio4C with AC or CC genotypes (rs6667202).

The inclusion criteria for the Perio4C group were the presence of true periodontal pockets and radiographic bone loss in patients up to 35 years of age; the presence of at least eight teeth with probing depth (PS) $\geq 5 \mathrm{~mm}$ (of which two teeth must have $\mathrm{PS} \geq 7 \mathrm{~mm}$ ) and bleeding on probing in 3 teeth not contiguous to the first molars and incisors; the presence of at least 20 teeth in the oral cavity.

The exclusion criteria were the presence of systemic changes (diabetes, heart disease, hepatitis) or use of medications (such as antibiotics, anti-inflammatory drugs, phenytoin, cyclosporine) that may influence the response to periodontal treatment in the six months before the study; periodontal treatment including subgingival instrumentation in the six months before the study; smoking habit, pregnancy, or lactation period.

\section{Collect of oral epithelial cell samples}

The patients rinsed with $5 \mathrm{~mL}$ of $3 \%$ dextrose for 1 minute. Afterward, the solution was centrifuged for $10 \mathrm{~min}$ at $3000 \mathrm{rpm}$ to sediment oral epithelial cells, as described by Trevilatto and Line ${ }^{23}, 2000$.

\section{DNA extraction and genotyping for rs6667202 SNP detection}

The oral epithelial cell samples were incubated at $55^{\circ} \mathrm{C}$ overnight in $1 \mathrm{~mL}$ of lysis solution [10mM Tris ( $\mathrm{pH} 8.0), 0.5 \%$ SDS, $5 \mathrm{mM}$ EDTA] with $10 \mu \mathrm{L}$ proteinase $\mathrm{K}(20 \mathrm{mg} / \mathrm{ml})$ (Sigma Chemical Co., St. Louis, MO, USA). After incubation, proteins and contaminants were removed by adding $500 \mu \mathrm{L}$ of $8 \mathrm{M}$ ammonium acetate solution and $1 \mathrm{mM}$ EDTA. The genomic DNA was precipitated in $540 \mu \mathrm{L}$ of isopropanol. The DNA was washed in $70 \%$ ethanol and resuspended in $50 \mu \mathrm{L} \mathrm{TE} \mathrm{pH} 8.0(10 \mathrm{mM}$ Tris and $1 \mathrm{mM}$ EDTA). In the end, the amount of purified DNA and its concentration were measured using a Nanodrop spectrophotometer (Thermo Scientific). 
The genotype for the rs6667202 variation was determined through DNA Polymerase Chain Reaction (PCR) in Real-Time, using the Taqman ${ }^{\circledR}$ system for allele discrimination (Applied Biosystems, Carlsbad, CA, USA). Primers and probes were designed using the Primer 3 program, allowing amplification of the regions where the variation is located. The TaqMan PCR was performed in a volume of 12 $\mu \mathrm{L}$ (3ng of DNA, 1X TaqMan master mix, $1 \times$ assay mix, 900nM of each primer, and 200nM of each probe) and distributed in 96 wells. The fluorescence of PCR amplification was detected using StepOne Plus (Applied Biosystems, Carlsbad, CA, USA) and analyzed with the manufacturer's software.

\section{Collect of GCF}

After removing the supragingival biofilm, the teeth were washed, and the area was isolated with cotton rollers and dried with air jets. GCF was collected through the insertion of filter paper strips (Periopaper, Oraflow) into the periodontal pocket or gingival sulcus for 15 seconds. Samples were collected from four sites in each patient, two sites with pockets of $P D \geq 7 \mathrm{~mm}$ and bleeding probing, two healthy sites with $\mathrm{PD} \leq 3 \mathrm{~mm}$ and no bleeding probing. The collected fluid volume was measured with a calibrated device for measurement of gingival fluid (Periotron 8000, Oraflow). The strips were stored in 400 $\mu$ l of phosphate-buffered saline (PBS) with 0.05\% Tween-20. Strips contaminated with visible blood were discarded, and a new sample was collected after 30 seconds. All samples were stored at $-80^{\circ} \mathrm{C}$ and remained under these conditions until the time of analysis.

\section{Immunoenzymatic Analysis}

Aliquots of each GCF sample were analyzed for IL-10 by Luminex/MAGpix technology. For this, the analyzes were performed in 96-well plates with the aid of high-sensitivity panels (HSCYTOMAG 60K, Millipore Corporation, Billerica, MA, USA), following the manufacturer's instructions. Briefly, the wells were washed with wash buffer, and aspirated and exclusive microspheres conjugated to monoclonal antibodies against the analyte (IL-10) were added. Samples and reagents for the standard curve were pipetted into the wells and incubated overnight at $4^{\circ} \mathrm{C}$. Then, the wells were washed, and a mixture of secondary antibodies was added. After incubation for 1 hour, the final detection was done through a third fluorescent marker, Streptavidin-Phycoerythrin, bound to the detection antibody. The concentration of IL-10 was expressed in $\mathrm{pg} / \mathrm{mL}$. Samples with quantification below the detection limit of the analysis were recorded as "zero", and samples above the limit of quantification of the standard curve were recorded with a value equal to the curve's highest value.

\section{Statistical Analyses}

The data from the immunoenzymatic assay were analyzed for normality by the Shapiro-Wilk test, and a comparison of IL-10 levels between groups was performed using the t-Student test. All tests were performed considering the significance level of $5 \%$.

\section{Results}

Table 1 shows the clinical and demographic data of Perio4C patients who participated in the study, indicating a similarity between the groups ( $p>0.05)$. 
Table 1. Demographic and clinical full mouth data of study participants.

\begin{tabular}{lcc}
\hline Characteristics & AA Group & AC Group \\
\hline Age (years) & $34.6 \pm 4.5$ & $34.4 \pm 4.4$ \\
\hline Gender (M/F) & $5 / 10$ & $4 / 11$ \\
\hline Plaque Index (\%) & $21.3 \pm 6.5$ & $24.4 \pm 6.4$ \\
\hline Bleeding Index (\%) & $26.8 \pm 9.0$ & $22.4 \pm 7.9$ \\
\hline Probing depth (mm) & $2.5 \pm 0.5$ & $2.2 \pm 0.2$ \\
\hline Probing Attachment Loss $(\mathrm{mm})$ & $5.1 \pm 1.1$ & $5.6 \pm 0.9$ \\
\hline
\end{tabular}

There was no significant difference between groups (Student's T and Chi-square tests, $p>0.05$ ).

* Indicates the statistical difference between groups.

Figure 1 shows the mean and standard deviation for shallow (1.A) and deep (1.B) pockets in the SNP + and SNP - groups. Regarding IL-10 levels, in shallow pockets, there was no significant difference in IL-10 GCF concentration between SNP + and SNP - groups $(0.05 \pm 0.04$ and $0.15 \pm 0.32$, respectively, p>0.05 - Figure 1.A). However, in deep pockets, the presence of rs6667202 SNP (SNP + Group) was associated with lower levels of IL-10 levels in the GCF when compared to the individuals with AC or CC genotypes (SNP - Group) (0.005 \pm 0.003 and $0.01 \pm 0.01$, respectively, $p=0.03$ Figure 1.B). Thus, based on the fact that in the deep pockets, the AC or CC genotypes had a higher level of IL-10 when compared to AA genotype Perio4C individuals, the protective character of the ancestral $\mathrm{C}$ allele for this disease could be firstly explained.

A

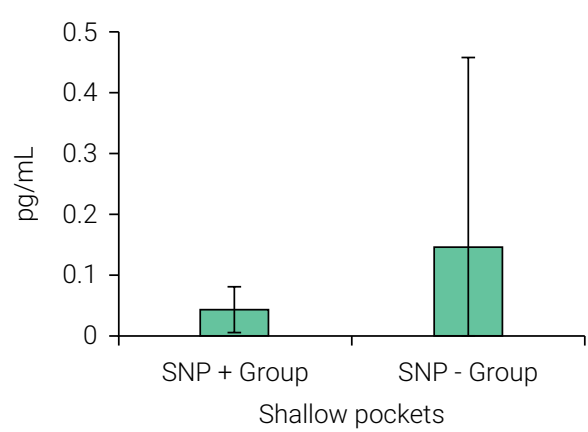

B

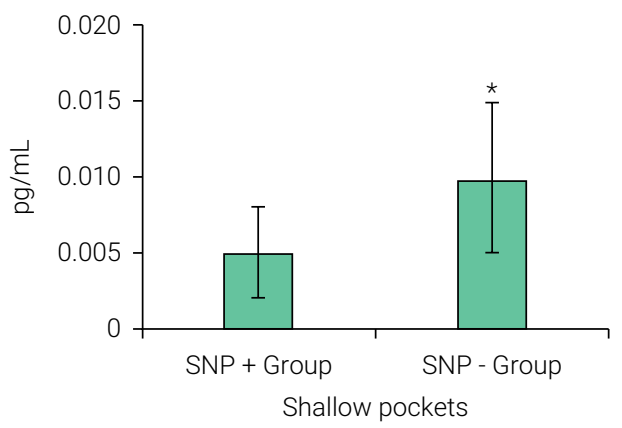

Figure 1. Levels of IL-10 in the GCF in shallow pockets $(P D \leq 3 \mathrm{~mm})(A)$ and deep pockets $(P D \geq 7 \mathrm{~mm})$ (B) of Perio4C AA genotype (rs6667202) patients (SNP + Group) and Perio4C AC or CC genotype (rs6667202) patients (SNP - Group).

\section{Discussion}

Although the prevalence of Perio4C appears to be exceptionally low in developed countries ${ }^{24}$, less developed regions show a higher frequency of this disease, where up to $5 \%$ of the population could be affected ${ }^{25,26}$. Epidemiological studies indicate that the prevalence of Perio4C can reach $5.5 \%$ of young individuals in the Brazilian population, and among young people aged $29-34$ years, this prevalence can reach $9.9 \% 25$. These percentages show that, especially in Brazil, there is a greater urgency to meet the 
demands caused by Perio4C. However, with this higher proportion of affected subjects, achieving a large population is always a difficult deal in research, especially with rigid inclusion criteria. Some previous studies have been done to identify one or more

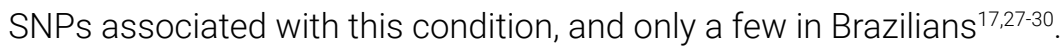

Traditionally, genetic research in periodontics has focused on identifying specific genetic changes, mainly from SNPs, presented as risk factors for periodontitis ${ }^{14,15,30}$. Despite these efforts, the genetic factors contributing to periodontal diseases' pathogenesis, especially Perio4C, are not yet fully defined ${ }^{29}$. In this sense, the current evidence suggests that Perio4C is polygenic, like other complex diseases, with the involvement of multiple genes with little effect (usually more than 100 genes for complex diseases) ${ }^{27}$.

Some studies have recently used a broader or large-scale assessment approach, the genome-wide association studies (GWAS), reporting the association of polymorphisms in genes hitherto not associated with Perio4C but which need their validation in independent populations ${ }^{14,17,28}$. Using this approach, Schaefer et al. ${ }^{28}$ (2010) conducted the first GWAS study in Perio4C, involving German individuals. It was observed that the genetic variant rs1537415 in the GLT6D1 gene, which indirectly controls the differentiation of Th2 cells, could be related to Perio4C. In the same context, rs1333048 SNP, in the CDKN2B antisense RNA 1 (ANRIL) gene, regulates mechanisms associated with colonization of the subgingival biofilm by periodontopathogens, also were associated with Perio4C in a European population ${ }^{30}$. In 2013, Schaefer et al. ${ }^{17}$ (2013) showed an association between rs6667202 IL10 gene SNP and Perio4C in a German-Austrian cohort. However, this association was not significant in some geographically close populations, as in the Dutch cohort. These results indicate the importance of validating SNPs for specific populations, once different regions could present a different genetic background.

The Brazilian population alone is highly miscegenated, resulting in genetic heterogeneity compared to other countries. Thus, our group conducted a study aiming to identify if there could be any association between the SNPs previously reported and the Perio4C in Brazilians. We found that the only SNP previously associated with Perio4C that showed to be significant to this population was the IL 10 SNP, rs6667202, and not GLT6D1 rs1537415 and ANRIL rs1333048 SNPs ${ }^{16}$, unlike what was found in other populations $^{17,28,30}$. Meanwhile, the populational association between SNP and any disease relies on other aspects - how the SNP could modify etiologic patterns and clinically impact disease occurrence. In this effort, this study assessed the local IL-10 levels in Perio4C affected-subjects to confirm rs6667202 functionality.

Genetic changes may have a direct influence on the immune response of patients affected by Perio4C ${ }^{13}$. These statements can be observed in studies that seek to understand the functionality of genetic variants, that is, how these variations can influence cellular functioning and/or alter microbiological colonization. No functional analysis had been performed to understand better the relationship between the SNP rs6667202 and the production of interleukin-10. Since this SNP is in a regulatory region of the IL-10 gene, we can presume an altered production of this cytokine when this SNP is present. When we analyze the results of this study, we notice an altered production of IL-10 in the SNP + Group, which presents the AA genotype compared to 
the SNP - Group, presenting AC or CC genotype ( $0.005 \pm 0.003$ and $0.01 \pm 0.01$, respectively, $p=0.03$ - Figure 1.B). This fact reinforces the protective character of the ancestral $C$ allele. It corroborates with the previous findings made by Taiete et al. ${ }^{16}$ (2019), where this allele was detected less frequently in Perio4C patients since, in this study, the AA group had a lower dosage of this anti-inflammatory cytokine. However, this result was dependent on pocket strata, i.e., IL-10 levels in GCF were differentially modulated by rs6667202 SNP in deep and shallow pockets.

In shallow pockets, there was no significant difference in IL-10 production between SNP + and SNP - groups (0.05 \pm 0.04 and 0.15 \pm 0.32 , respectively, p>0.05 - Figure 1.A). It could be related to the fact that a symbiotic community is colonizing the subgingival environment in healthy sites and does not pose a challenge to the host immune system $^{20,31,32}$. When we analyze the shallow pockets in Figure 1, we see a significant difference between the mean values of IL-10 in both groups. However, the standard deviation was high, especially for the SNP - group; that is, there was a significant impact of the data variability on the results. It may be related to the presence of pathogens and the onset of a dysbiotic environment at the GCF collected sites of some patients, who have not yet shown clinical changes at these sites despite already having an altered inflammatory response.

The same thought could be attributed to deep pockets, where a mature and dysbiotic biofilm instigates the host response, but now leading to clinical alterations. It is well known that IL-10 is highly expressed in inflamed periodontal tissues and acts as the main inhibitor of pro-inflammatory and harmful cytokines in the context of periodontal destruction. It can downregulate the synthesis of pro-inflammatory cytokines and chemokines, such as interleukin 1 (IL-1), IL-6, TNF-a, nitric oxide, gelatinase, and collagenase $^{33}$, and upregulate the synthesis of IL-1 and TNF-a when specifically neutralized $^{34}$. In addition, IL-10 has a major impact on the adaptive immune response. It led $\mathrm{B}$ cells to differentiate and proliferate, reducing apoptosis of immune cells and stimulating Cd8+ and natural killer cells ${ }^{18,19}$. Thus, it could be suggested that when the subject presents the AA genotype, IL-10 production is reduced, altering the immune response against microbial aggression in periodontal pockets, and inducing more severe destruction.

Corroborating to this pathological pathway, several immunological and microbiological factors have been strongly associated with Perio4C, which can be associated with the occurrence and the low response of conventional periodontal treatment. In addition to the study of Casarin et al. ${ }^{20}$ (2010) that showed reduced IL-10 levels in GCF associated with low production of Immunoglobulin G (IgG) against Pg and $\mathrm{Aa}$ in Perio4C patients, other studies have also suggested a reduction in IL-10 expression both in GCF, blood serum, and gingival tissue ${ }^{21,22}$ in these individuals. These findings can probably be related to polymorphisms associated with Perio4C, some of them described in previous studies ${ }^{15,35}$. For example, the haplotype ATA of IL-10, as a "Iow interleukin-10 producer", was proved to be a risk indicator for Perio4C ${ }^{35}$, emphasizing the importance of IL-10 in Perio4C etiology.

At the moment, this discovery indicates a better understanding of the pathogenesis of this condition. It soon will instigate other in vitro analysis to better comprehends it cell behavior and its relationship with the presence of pathogens in Perio4C, and with a 
larger population, to widely confirm its role on disease etiopathogenic aspects, allowing the identification of patients with greater susceptibility and establishing an individualized periodontal support therapy according to patient's biological characteristics.

We conclude that IL 10 rs6667202 SNP is associated with lower production of IL-10 in crevicular fluid, potentially affecting this disease progression and reinforcing the protective character of the ancestral $\mathrm{C}$ allele evaluated before.

\section{References}

1. Armitage GC. Development of a classification system for periodontal diseases and conditions. Ann Periodontol. 1999 Dec;4(1):1-6. doi: 10.1902/annals.1999.4.1.1.

2. Albandar JM. Aggressive and acute periodontal diseases. Periodontol 2000. 2014 Jun;65(1):7-12. doi: 10.1111/prd.12013.

3. Caton JG, Armitage G, Berglundh T, Chapple ILC, Jepsen S, Kornman KS, et al. A new classification scheme for periodontal and peri-implant diseases and conditions - Introduction and key changes from the 1999 classification. J Periodontol. 2018 Jun;89 Suppl 1:S1-S8. doi:10.1002/JPER.18-0157

4. Tonetti MS, Mombelli A. Early-onset periodontitis. Ann Periodontol. 1999 Dec;4(1):39-53. doi: 10.1902/annals.1999.4.1.39.

5. Christersson LA, Slots J, Rosling BG, Genco RJ. Microbiological and clinical effects of surgical treatment of localized juvenile periodontitis. J Clin Periodontol. 1985 Jul;12(6):465-76. doi: 10.1111/j.1600-051x.1985.tb01382.x.

6. Deas DE, Mealey BL. Response of chronic and aggressive periodontitis to treatment. Periodontol 2000. 2010 Jun;53:154-66. doi:10.1111/j.1600-0757.2009.00334.x. Review.

7. Scharf S, Wohlfeil M, Siegelin Y, Schacher B, Dannewitz B, Eickholz P. Clinical results after nonsurgical therapy in aggressive and chronic periodontitis. Clin Oral Investig. 2014;18(2):453-60. doi:10.1007/s00784-013-1013-4.

8. Teughels W, Dhondt R, Dekeyser C, Quirynen M. Treatment of aggressive periodontitis. Periodontol 2000. 2014 Jun;65(1):107-33. doi: 10.1111/prd.12020.

9. Guerrero A, Griffiths GS, Nibali L, Suvan J, Moles DR, Laurell L, et al. Adjunctive benefits of systemic amoxicillin and metronidazole in non-surgical treatment of generalized aggressive periodontitis: a randomized placebo-controlled clinical trial. J Clin Periodontol. 2005 Oct;32(10):1096-107. doi: 10.1111/j.1600-051X.2005.00814.x.

10. Bastos MF, Lima JA, Vieira PM, Mestnik MJ, Faveri M, Duarte PM. TNF-alpha and IL-4 levels in generalized aggressive periodontitis subjects. Oral Dis. 2009 Jan;15(1):82-7. doi: 10.1111/j.1601-0825.2008.01491.x.

11. Duarte PM, da Rocha M, Sampaio E, Mestnik MJ, Feres M, Figueiredo LC, et al. Serum levels of cytokines in subjects with generalized chronic and aggressive periodontitis before and after non-surgical periodontal therapy: a pilot study. J Periodontol. 2010 Jul;81(7):1056-63. doi: 10.1902/jop.2010.090732.

12. Shaddox L, Wiedey J, Bimstein E, Magnuson I, Clare-Salzler M, Aukhil I, et al. Hyper-responsive phenotype in localized aggressive periodontitis. J Dent Res. 2010 Feb;89(2):143-8. doi: 10.1177/0022034509353397.

13. Loos BG, Papantonopoulos G, Jepsen S, Laine ML. What is the contribution of genetics to periodontal risk? Dent Clin North Am. 2015 Oct;59(4):761-80. doi: 10.1016/j.cden.2015.06.005. 
14. Loos BG, John RP, Laine ML. Identification of genetic risk factors for periodontitis and possible mechanisms of action. J Clin Periodontol. 2005;32 Suppl 6:159-79. doi: 10.1111/j.1600-051X.2005.00806.x.

15. Nibali L, Pelekos G, D’Aiuto F, Chaudhary N, Habeeb R, Ready D, Parkar M, et al. Influence of il-6 haplotypes on clinical and inflammatory response in aggressive periodontitis. Clin Oral Investig. 2013 May;17(4):1235-42. doi: 10.1007/s00784-012-0804-3.

16. Taiete T, Casati MZ, Stolf CS, Corrêa MG, Santamaria MP, Andere NMRB, et al. Validation of reported GLT6D1 (rs1537415), IL 10 (rs6667202), and ANRIL (rs1333048) single nucleotide polymorphisms for aggressive periodontitis in a Brazilian population. J Periodontol. 2019 Jan;90(1):44-51. doi: 10.1002/JPER.18-0071.

17. Schaefer AS, Bochenek G, Manke T, Nothnagel M, Graetz C, Thien A, et al. Validation of reported genetic risk factors for periodontitis in a large-scale replication study. J Clin Periodontol. 2013 Jun;40(6):563-72. doi: 10.1111/jcpe.12092.

18. Garlet GP. Destructive and protective roles of cytokines in periodontitis: a re-appraisal from host defense and tissue destruction viewpoints. J Dent Res. 2010 Dec;89(12):1349-63. doi: 10.1177/0022034510376402.

19. Garlet GP, Cardoso CR, Campanelli AP, Martins W Jr, Silva JS. Expression of suppressors of cytokine signaling in diseased periodontal tissues: a stop signal for disease progression? J Periodontal Res. 2006 Dec;41(6):580-4. doi: 10.1111/j.1600-0765.2006.00908.x.

20. Casarin RC, Ribeiro Edel P, Mariano FS, Nociti FH Jr, Casati MZ, Gonçalves RB. Levels of Aggregatibacter actinomycetemcomitans, Porphyromonas gingivalis, inflammatory cytokines and species-specific immunoglobulin $\mathrm{G}$ in generalized aggressive and chronic periodontitis. J Periodontal Res. 2010 Oct;45(5):635-42. doi: 10.1111/j.1600-0765.2010.01278.x.

21. Teles RP, Gursky LC, Faveri M, Rosa EA, Teles FR, Feres M, et al. Relationships between subgingival microbiota and GCF biomarkers in generalized aggressive periodontitis. J Clin Periodontol. 2010 Apr;37(4):313-23. doi: 10.1111/j.1600-051X.2010.01534.X.

22. Mattuella LG, Campagnaro MB, Vargas AE, Xavier LL, Oppermann RV, Chies JA, et al. Plasma cytokines levels in aggressive and chronic periodontitis. Acta Odontol Scand. 2013 MayJul;71(3-4):683-8. doi: 10.3109/00016357.2012.715191.

23. Trevilatto PC, Line SR. Use of buccal epithelial cells for PCR amplification of large DNA fragments. J Forensic Odontostomatol. 2000 Jun;18(1):6-9.

24. Albandar JM, Tinoco E. Global epidemiology of periodontal diseases in children and young persons. Periodontol 2000. 2002;29:153-76. doi: 10.1034/j.1600-0757.2002.290108.x.

25. Susin C, Albandar JM. Aggressive periodontitis in an urban population in southern Brazil. J Periodontol. 2005 Mar;76(3):468-75. doi: 10.1902/jop.2005.76.3.468.

26. Susin C, Haas AN, Albandar JM. Epidemiology and demographics of aggressive periodontitis. Periodontol 2000. 2014 Jun;65(1):27-45. doi: 10.1111/prd.12019.

27. Laine ML, Crielaard W, Loos BG. Genetic susceptibility to periodontitis. Periodontol 2000. 2012 Feb;58(1):37-68. doi: 10.1111/j.1600-0757.2011.00415.x.

28. Schaefer AS, Richter GM, Nothnagel M, Manke T, Dommisch H, Jacobs G, et al. A genome-wide association study identifies GLT6D1 as a susceptibility locus for periodontitis. Hum Mol Genet. 2010 Feb 1;19(3):553-62. doi: 10.1093/hmg/ddp508.

29. Vieira AR, Albandar JM. Role of genetic factors in the pathogenesis of aggressive periodontitis. Periodontol 2000. 2014 Jun;65(1):92-106. doi: 10.1111/prd.12021.

30. Schaefer AS, Richter GM, Groessner-Schreiber B, Noack B, Nothnagel M, El Mokhtari NE, et al. Identification of a shared genetic susceptibility locus for coronary heart disease and periodontitis. PLoS Genet. 2009;5(2):e1000378. doi:10.1371/journal.pgen.1000378. 
31. Cortelli JR, Cortelli SC, Jordan S, Haraszthy VI, Zambon JJ. Prevalence of periodontal pathogens in Brazilians with aggressive or chronic periodontitis. J Clin Periodontol. 2005 Aug;32(8):860-6. doi: 10.1111/j.1600-051X.2005.00777.x.

32. Könönen E, Müller HP. Microbiology of aggressive periodontitis. Periodontol2000. 2014 Jun;65(1):46-78. doi: 10.1111/prd.12016.

33. Houri-Haddad Y, Soskolne WA, Halabi A, Shapira L. IL-10 gene transfer attenuates P. gingivalisinduced inflammation. J Dent Res. 2007 Jun;86(6):560-4. doi: 10.1177/154405910708600614.

34. Marinou I, Healy J, Mewar D, Moore DJ, Dickson MC, Binks MH, et al. Association of interleukin-6 and interleukin-10 genotypes with radiographic damage in rheumatoid arthritis is dependent on autoantibody status. Arthritis Rheum. 2007 Aug;56(8):2549-56. doi: 10.1002/art.22814.

35. Reichert S, Machulla HK, Klapproth J, Zimmermann U, Reichert Y, Gläser CH, et al. The interleukin-10 promoter haplotype ATA is a putative risk factor for aggressive periodontitis. J Periodontal Res. 2008 Feb;43(1):40-7. doi: 10.1111/j.1600-0765.2007.00992.x. 